\title{
Liver Biopsy in Liver Transplant Recipients
}

\author{
Thuong G. Van Ha, M.D. ${ }^{1}$
}

Liver biopsy has been used in the assessment of the nature and course of liver diseases and to monitor treatments. In nontransplanted patients, liver biopsies have been well described. Less has been written on the biopsies of transplanted livers. In the liver transplant population, liver biopsy remains the "gold standard" for the diagnosis of rejection. The transplanted liver has additional considerations that can make biopsy less routine and more challenging.

KEYWORDS: Liver biopsy, liver transplant, percutaneous liver biopsy, transjugular liver biopsy

Objectives: Upon completion of this article, the reader should be able to identify the special considerations given to biopsy of the transplanted liver and the techniques used in liver biopsy.

Accreditation: Tufts University School of Medicine (TUSM) is accredited by the Accreditation Council for Continuing Medical Education to provide continuing medical education for physicians.

Credit: TUSM designates this educational activity for a maximum of 1 Category 1 credit toward the AMA Physicians Recognition Award. Each physician should claim only those credits that he/she actually spent in the activity.

Liver biopsy is used to make a diagnosis, to assess disease severity, and to monitor treatments of liver diseases in nontransplanted patients as well as in liver transplant recipients. Liver biopsy can provide a diagnosis in $\sim 90 \%$ of patients with unexplained abnormal liver function tests. ${ }^{1}$ In transplant recipients, liver biopsy is the gold standard for diagnosis of acute rejection. ${ }^{2,3}$

Neuberger and associates advocate protocol liver biopsy for transplant recipients, both early and late. ${ }^{4} \mathrm{~A}$ protocol biopsy is one that is made according to guidelines rather than in response to the patient's clinical status. Early protocol biopsy is usually performed 7 days after transplantation because liver function tests are insufficient for the diagnosis and assessment of severity of rejection. Graft function is better preserved if rejection is treated early and the histology of the allograft allows better management. Late protocol biopsies are performed after 6 months. Information from late biopsy enables physicians to distinguish between normal and abnormal graft histology, interpret liver function tests, adjust immunosuppressive doses, and recognize current disease.

A specimen size of $1.5 \mathrm{~cm}$ is needed for a diagnosis of diffuse liver disease to be made. In addition, six to eight portal triads must be included in the sample because the extent of injury may vary among the triads. ${ }^{5}$ Available needles, whether thin bore, spring loaded, or of suction type, usually provide adequate specimens. ${ }^{6}$

Currently, there are several techniques that can be used to obtain liver tissue, including percutaneous liver biopsy or aspiration, transjugular liver biopsy, seldom used laparoscopic liver biopsy, and surgical open liver biopsy. The open biopsy is usually performed when the patient's abdomen has to be entered for another procedure rather than as the primary procedure. Laparoscopic liver biopsy, although useful in the diagnosis of

Transplant Interventions; Editor in Chief, Peter R. Mueller, M.D.; Guest Editor, Ravi Murthy, M.D. Seminars in Interventional Radiology, volume 21, number 4, 2004. Address for correspondence and reprint requests: Thuong G. Van Ha, M.D., Department of Radiology, Section of Interventional Radiology, The University of Chicago, 5841 South Maryland Avenue, MC 2026, Chicago, IL 60615. ${ }^{1}$ Department of Radiology, Section of Interventional Radiology, The University of Chicago, Chicago, Illinois. Copyright (C) 2004 by Thieme Medical Publishers, Inc., 333 Seventh Avenue, New York, NY 10001, USA. Tel: +1(212) 584-4662. 0739-9529,p;2004,21,04,271,274,ftx,en;sir00266x. 
peritoneal disease and staging of cancer, ${ }^{7}$ has fallen out of favor because there are less invasive procedures of percutaneous and transjugular liver biopsy.

Although the general principles of liver biopsy are the same, additional considerations must be given to transplant recipients. Indications for liver biopsy in this population include evaluation for rejection as well as to guide therapy.

\section{PERCUTANEOUS LIVER BIOPSY}

In patients with native livers, whether or not ultrasonography is used during liver biopsy is debatable, although the need to assess the liver prior to biopsy has been recommended. ${ }^{6}$ Routine use of ultrasound guidance in nontransplanted livers has not proved to reduce complications, to be more cost-effective, or have a better diagnostic yield. ${ }^{8-10}$ In the grafted patient, ultrasonography defines the anatomy, identifies abnormalities such as ascites, and allows visualization of a safe path into the transplant. At times, as in split liver or left lateral segment transplantation, ultrasound or computed tomography (CT) guidance is needed to avoid passage of needle through other organs such as interposed bowel. At the University of Chicago, we routinely use ultrasound guidance for percutaneous liver biopsy. Occasionally, CT guidance is required.

Contraindications to percutaneous liver biopsy include uncooperative patients, uncorrectable coagulopathy, thrombocytopenia (platelets $<50,000 / \mathrm{mm}^{3}$ ), ascites, and infections in the right pleural cavity or right upper quadrant of the abdomen.

The types of needles used in percutaneous liver biopsies can be divided into the suction types, such as the Menghini (Baxter Health Care, Deerfield, IL) or Jamshidi (Allegiance, McGaw Park, IL) needles, or the cutting types, such as the Tru-Cut needle (Baxter Health Care, Deerfield, IL). The cutting needle is associated with increased risks of bleeding, as with larger bore needles. ${ }^{11,12}$ Another type of biopsy needle is the spring-loaded cutting needle, which minimizes the procedure time and liver dwell time and therefore decreases the risk of bleeding. A cutting needle is preferred in cirrhotic livers, as suction needles tend to fragment the fibrotic liver parenchyma. ${ }^{13,14}$

Percutaneous liver biopsy can be performed on an outpatient basis, if various conditions are met, as recommended by the American Gastroenterological Association guidelines. ${ }^{15}$ Janes and Lindor retrospectively reviewed 405 patients who underwent liver biopsy as outpatients. Three percent were hospitalized with complications. ${ }^{16}$ Of this group, 38\% were admitted with localized pain, 38\% with orthostatic hypotension, $8 \%$ with both pain and hypotension, $8 \%$ with peritoneal signs, and $8 \%$ with lightheadedness. Five patients had bleeding identified radiographically, but only two pa- tients required blood transfusions. All complications were identified within 3 hours after biopsy. On the basis of this article, it would seem judicious to observe patients for at least 3 hours after liver biopsy.

Complications of percutaneous liver biopsy are rare and most are manifest within 24 hours of the procedure. $^{12,15,16}$ Minor complications include localized pain and mild, transient hypotension due to vasovagal reaction. Persistent or severe pain might indicate a more serious event such as hemorrhage. Although rare, bleeding is the most serious complication of percutaneous liver biopsy. Bleeding can be localized, subcapsular, or intraperitoneal. The last complication usually results from laceration or penetrating injury to the hepatic arterial or portal venous system. ${ }^{12,17}$ As mentioned previously, cutting-type needles (not spring loaded) and larger bore needles are associated with higher bleeding complications. ${ }^{10}$ Other risk factors for hemorrhage include cirrhosis, older age, and presence of liver tumors. ${ }^{12,16}$

At our institution, if a significant bleeding complication is identified, the patient is admitted and fluid resuscitation is started. At the same time, surgical and interventional radiology consultations are obtained. For a patient with a graft, the transplant service is notified. Blood transfusions might be necessary. Aggressive management is typical to avert disastrous consequences. If a patient remains hemodynamically unstable, angiography and embolization should be considered. Emergent surgical exploration may be required.

Small, localized bleeding, whether intrahepatic or subcapsular, generally abates with conservative medical management. Other complications include bacteremia, pneumothorax, abscess, and bile peritonitis. ${ }^{6}$ There is no evidence to support the use of antibiotics for routine liver biopsy, even though transient bacteremia, usually not clinically significant, can occur. ${ }^{18}$ However, septicemia can occur in patients with biliary obstruction and cholangitis. In transplant recipients, there is an increased risk of septicemia in patients with cholodochojejunostomy. ${ }^{3}$ In this setting antibiotics might be warranted. Hemobilia is a rare complication that is manifest as a triad of pain, gastrointestinal bleeding, and jaundice. ${ }^{12,19}$ This complication can be successfully treated with embolization. The mortality rate in percutaneous liver biopsy ranges from 1 in 10,000 to 1 in $12,000^{6,11,12,17}$

\section{TRANSJUGULAR LIVER BIOPSY}

Transjugular liver biopsy utilizes the transvenous route to obtain liver samples. This reduces the risk of bleeding. Indications are listed in Table 1. Typically, the right internal jugular vein is punctured and the right hepatic vein is selected. A right hepatic venogram is performed to confirm position and assess venous patency. A stiff curved sheath is then introduced into the central aspect 


\section{Table 1 Indications for Transjugular Liver Biopsy}

Severe coagulopathy
Thrombocytopenia
Massive ascites
Morbid obesity
Additional procedure warranted (e.g., wedged hepatic pressure
measurements)
Failure of percutaneous liver biopsy

of the right hepatic vein and samples are obtained with a biopsy needle. At our institution, we use the 19-gauge Quick-Core biopsy needle (Cook, Bloomington, IN) and obtain two or three samples. Although others have stated a 30 to 60 minutes duration for the procedure, ${ }^{6}$ our experience indicates a much shorter duration of 15 to 20 minutes unless additional procedures are performed. Common additional procedures are hepatic venograms and wedged hepatic vein pressure measurements. Occasionally, transjugular liver biopsy is performed prior to transjugular intrahepatic portosystemic shunt (TIPS) placement. A histologic diagnosis can be made in $80-97 \%$ of procedures. ${ }^{20}$ Inadequate specimens usually result from tissue fragmentation.

In patients with liver transplants, transjugular liver biopsy has been reported to be safe and efficacious. ${ }^{21}$ In a series of 124 biopsies of 105 patients in the early post-transplantation period, Azouley and associates reported a technical success rate of $87 \%$ with adequate tissue for diagnosis in most instances. ${ }^{22}$ In addition, the clinical management was influenced by the results of the biopsies in $65 \%$ of cases, including the initiation of antirejection therapy in 59\% of the cases. In this series, there were more technical failures associated with patients with a preserved native inferior vena cava. Lerut et al reported a series of patients with grafts who underwent TIPS. ${ }^{23}$ These authors stressed that TIPS placement could be technically difficult in some patients, particularly those with piggy-back, cavocaval anastomoses because of difficulty in cannulation of the allograft hepatic and portal vein. It can be inferred from their experience that transjugular liver biopsies in these patients would pose technical difficulties as well.

In our experience of 100 consecutive transjugular liver biopsies, including 42 in patients with transplants, compared with 110 consecutive cases of image-guided percutaneous liver biopsies, including 82 in transplanted liver, our technical success rates were 99\% and 95\% for the respective techniques. ${ }^{24}$ Adequate tissues were obtained in $98 \%$ and $96 \%$, respectively. Our high success rate in transjugular liver biopsies might be due in part to the fact that our patients with transplants received the conventional surgical approach with caval replacement.

Complications of transjugular liver biopsy occur in 1.3 to $20 \%$ of cases and the mortality ranges from 0.1 to $0.5 \% .{ }^{20,25}$ Bleeding complications usually occur in capsular perforation. In fact, capsular perforation can occur in up to $4.4 \%$ of transjugular liver biopsies. ${ }^{26,27} \mathrm{In}$ liver transplant recipients, there are no large reported series documenting complications and mortality. In our series, we had one bleeding complication out of 100 cases, 42 of which were in transplanted livers. ${ }^{24}$

Although the American Society of Clinical Oncology recommends a minimum platelet count for percutaneous liver biopsy of $50 \times 10^{9} / \mathrm{L}$, there is no recommended minimum platelet count for the transjugular route. Wallace and associates reported 51 transvenous liver biopsies in 50 patients with severe thrombocytopenia and hematologic malignancy whose platelets were $30 \times 10^{9} / \mathrm{L}$ or lower. ${ }^{28}$ The mean platelet count was $17 \times 10^{9}$ and a mean of 11 units of platelets were transfused. The postbiopsy mean platelet count was $38 \times 10^{9}$ but was $30 \times 10^{9}$ or lower in 24 patients. The authors concluded that a threshold platelet count for safe biopsy is below $30 \times 10^{9}$.

In conclusion, liver biopsy in transplant recipients remains the gold standard for the diagnosis of rejection. Image-guided percutaneous liver biopsy in this population is safe and efficacious if there are no contraindications. If there are, transjugular liver biopsy should be considered.

\section{REFERENCES}

1. Hultcrantz R, Gabrielsson N. Patients with persistent elevation of aminotransferases: investigation with ultrasonography, radionuclide imaging and liver biopsy. J Intern Med 1993;233:7-12

2. Colonna JO II, Brems JJ, Goldstein LI, et al. The importance of percutaneous liver biopsy in the management of the liver transplant recipient. Transplant Proc 1988;20(suppl 1):682684

3. Bubak ME, Porayko MK, Krom RAF, Wiesner RH. Complications of liver biopsy in liver transplant patients: increased sepsis associated with choledochojejunostomy. Hepatology 1991;14:1063-1065

4. Neuberger J, Wilson P, Adams D. Protocol liver biopsies: the case in favour. Transplant Proc 1998;30:1497-1499

5. General principles. In: Lee RG. Diagnostic Liver Pathology. St Louis: Mosby-Year Book; 1994:1-21

6. Bravo A, Sheth SG, Chopra S. Current concepts: liver biopsy. N Engl J Med 2001;344:495-500

7. Vargas C, Jeffers LJ, Bernstein D, et al. Diagnostic laparoscopy: a 5-year experience in a hepatology training program. Am J Gastroenterol 1995;90:1258-1262

8. Vautier G, Scott B, Jenkins D. Liver biopsy: blind or guided? BMJ 1994;309:1455-1456

9. Pasha T, Gabriel S, Therneau T, Dickson ER, Lindor KD. Cost-effectiveness of ultrasound guided liver biopsy. Hepatology 1998;27:1220-1226

10. Younossi ZM, Teran JC, Ganiats TG, Carey WD. Ultrasound-guided liver biopsy for parenchymal liver disease: an economic analysis. Dig Dis Sci 1998;43:46-50 
11. McGill DB, Rakela J, Zinsmeister AR, Ott BJ. A 21-year experience with major hemorrhage after percutaneous liver biopsy. Gastroenterology 1990;99:1396-1400

12. Piccinino F, Sagnelli E, Pasquale G, Giusti G. Complications following percutaneous liver biopsy: a multicentre retrospective study on 68,276 biopsies. J Hepatol 1986;2:165-173

13. Bateson MC, Hopwood D, Duguid HL, Bouchier IA. A comparative trial of liver biopsy needles. J Clin Pathol 1980; 33:131-133

14. Colombo M, Del Ninno E, de Franchis R, et al. Ultrasoundassisted percutaneous liver biopsy: superiority of the Tru-Cut over the Menghini needle for diagnosis of cirrhosis. Gastroenterology 1988;95:487-489

15. Jacobs WH, Goldberg SB. Statement on outpatient percutaneous liver biopsy. Dig Dis Sci 1989;34:322-323

16. Janes $\mathrm{CH}$, Lindor $\mathrm{KD}$. Outcome of patients hospitalized for complications after outpatient liver biopsy. Ann Intern Med 1993;118:96-98

17. Van Thiel DH, Gavaler JS, Wright H, Tzakis A. Liver biopsy: its safety and complications as seen at a liver transplant center. Transplantation 1993;55:1087-1090

18. Reddy KR, Schiff ER. Complications of liver biopsy. In: Taylor MB, ed. Gastrointestinal Emergencies, 2nd ed. Baltimore: Williams \& Wilkins; 1997:959-968

19. Lichtenstein DR, Kim D, Chopra S. Delayed massive hemobilia following percutaneous liver biopsy: treatment by embolotherapy. Am J Gastroenterol 1992;87:1833-1838
20. Lebrec D, Goldfarb G, Degott C, Rueff B, Benhamou JP. Transvenous liver biopsy: an experience based on 1000 hepatic tissue samplings with this procedure. Gastroenterology 1982;83:338-340

21. Snover DC, Freese DK, Sharp HL, et al. Liver allograft rejection: an analysis of the use of biopsy in determining the outcome of rejection. Am J Surg Pathol 1987;11:1-10

22. Azouley D, Raccuia JS, Rache B, Reynes M, Bismuth H. The value of early transjugular liver biopsy after liver transplantation. Transplantation 1996;61:406-409

23. Lerut JP, Goffette P, Molle G, et al. Transjugular intrahepatic portosystemic shunt after adult liver transplantation; experience in eight patients. Transplantation 1999;68:379-384

24. Lin BH, Van Ha T, Zeikus E, et al. Transjugular liver biopsy in patients with liver transplant and coagulopathies. AJR Am J Roentgenol 2001;176(suppl):60

25. McAfee JH, Keeffe EB, Lee RG, Rosch J. Transjugular liver biopsy. Hepatology 1992;15:726-732

26. Brenard R, Horsmans Y, Rahier J, Druez P, Descamps C, Geubel A. Transjugular liver biopsy: an experience based on 500 procedures. Acta Gastroenterol Belg 1997;60:138-141

27. Papatheodoridis GV, Patch D, Watkinson A, Tibballs J, Burroughs AK. Transjugular liver biopsy in the 1990s: a 2-year audit. Aliment Pharmacol Ther 1999;13:603-608

28. Wallace MJ, Narvios A, Lichtiger B, et al. Transjugular liver biopsy in patients with hematologic malignancy and severe thrombocytopenia. J Vasc Interv Radiol 2003;14:323-327 